\title{
Klinische Ernährungsmedizin
}

$\mathrm{D}$ ie Ernährung als prophylaktische und therapeutische Maßnahme ist eine wichtige Komponente im medizinischen Alltag. Zwar rückt heute die rasche Zunahme an Übergewichtigen immer stärker in unseren Fokus - die Adipositas ist bereits eine Epidemie, die fast nicht mehr einzudämmen ist. Die damit assoziierten Begleiterkrankungen und deren notwendigen Therapien führen zu einem enormen Anstieg der Gesundheitskosten, sie machen schätzungsweise etwa 10\% der Aufwendungen in unserem Gesundheitssystem aus.

Wesentlich weniger wahrgenommen und damit in ihrem Ausmaß drastisch unterschätzt wird jedoch die Mangelernährung oder Malnutrition. Schon bei ihrer Aufnahme in die Klinik zeigen - je nach Krankenhaus 20-60\% aller Patienten Zeichen einer Mangelernährung. Auch in der Klinik nehmen die Patienten oft noch ab. Zu häufig werden solche Risikopatienten allerdings nicht erfasst und erhalten daher auch keine entsprechende Ernährungstherapie. Dies ist nicht erstaunlich und Gründe dafür sind bekannt: Schon in der Ausbildung von Ärzten und Pflegenden ist die klinische Ernährung in den meisten Ländern unterrepräsentiert. Die Hochschulen haben oft keine offiziellen Lernziele für die klinische Ernährung, nur an wenigen Universitäten sind entsprechende Lehrstühle eingerichtet. Zudem verfügen nur wenige Krankenhäuser über spezielle Abteilungen oder Verantwortliche für Ernährungsmedizin. Dieses mangelnde Bewusstsein an der klinischen Ernährung hat zur Folge, dass gerade die Patienten mit einer Mangelernährung nicht optimal betreut werden.

Ein wichtiger Schritt zur Verbesserung dieser unbefriedigenden Situation könnte der Bericht des Europarats aus dem Jahr 2002 sein. Dieses Dokument formuliert wichtige Faktoren, die zu der unbefriedigenden Ernährungssituation in Kliniken beitragen und gibt gleichzeitig Empfehlungen zur Verbesserung. Als mangelhaft und damit verbesserungswürdig bewertet der Europarat die Zuweisung von Verantwortlichkeiten für die Organisation und den Einsatz der Ernährungstherapie, die Ausbildung des Klinikpersonals bezüglich Ernährungstherapien, den Einfluss der Patienten auf Verpflegungsangebote und Ernährungstherapien, die interdisziplinäre klinische Kooperation sowie die Unterstützung seitens der Klinik- und Heimverwaltung. Demnach besteht ein großer Handlungsbedarf in vielen Bereichen - angefangen von der Erfassung des Ernährungsstatus bei der Aufnahme der Patienten in die Klinik über den Einsatz einer perioperativen immunmodulierenden Ernährung bis hin zur optimierten enteralen Langzeiternährung, die zu Hause weitergeführt werden kann.
Mit dieser Schwerpunktausgabe des klinikarzt wollen wir das Interesse an der Ernährungsmedizin wecken. An speziellen Beispielen zeigen wir auf, wie wichtig die Ernährung ist und welche Vorteile sie für Patienten und Kostenträger haben kann. Denn in der Klinik ist die Mangelernährung - vor allem auch bei Tumorpatienten - ein zentrales, meist multifaktoriell verursachtes Problem. Die Malnutrition aber führt zu einer erhöhten Morbidität und Mortalität und ist oft ein nicht erkannter Grund für die Kostensteigerung im Gesundheitswesen. Daher sollten der Ernährungszustand sowie eine adäquate Ernährungstherapie als Bestandteil grundsätzlich in jedem Behandlungskonzept eines Patienten integriert sein. Es ist zu hoffen, dass in Zukunft die Bedeutung der Ernährung durch gezielte Schulung an Universitäten und Kliniken vermehrt bewusst wird, und die Ernährungsmedizin dann auch in der Praxis adäquat umgesetzt wird.

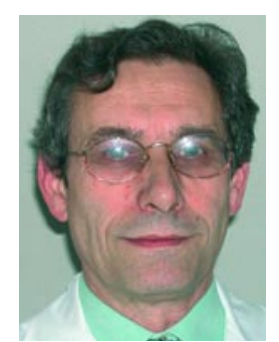

PD Dr. Rémy Meier, Liestal (Schweiz)

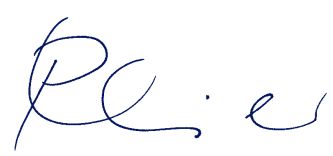

